\title{
ON DYNAMIC BIFURCATIONS IN CONTROL SYSTEMS
}

\author{
Fritz Colonius * Wolfgang Kliemann** \\ - Institute for Mathematics, University of Augsburg, \\ 86135 Augsburg, Germany, colonius@math.uni-augsburg.de \\ ** Department of Mathematics, Iowa State University, \\ Ames IA 50011/U.S.A., kliemann@iastate.edu
}

\begin{abstract}
We discuss controllability properties of control affine systems depending on a slowly varying parameter. Near a singular point, the control sets for fixed parameter values turn into subsets where controllability holds provided that the parameter variations are slow enough. Copyright ${ }^{\oplus} 2001$ IFAC
\end{abstract}

Keywords: controllability

\section{INTRODUCTION}

We consider the following family of control-affine systems depending on a real parameter $\alpha$ :

$$
\begin{aligned}
\dot{x}(t) & =f_{0}(\alpha, x(t))+\sum_{i=1}^{m} u_{i}(t) f_{i}(\alpha, x(t)), \\
u & =\left(u_{i}\right) \in \mathcal{U}=\left\{u: \mathbb{R} \rightarrow U \subset \mathbb{R}^{m}, \text { integrable }\right\},
\end{aligned}
$$

where $f_{0}, \ldots, f_{m}$ are smooth vector fields on $\mathbb{R}^{d}$ and the control range $U$ is convex and compact; the parameter $\alpha$ takes values in an interval $A=$ $[a, b) \subset \mathbb{R}$. We assume that unique solutions $\varphi\left(t, x_{0}, u, \alpha\right), t \in \mathbb{R}$, exist for all $x_{0}, u$, and $\alpha$. We will replace $\alpha$ by a time dependent, slowly varying parameter, and our goal is to discuss the changes in the controllability behavior as $\alpha$ evolves.

For simplicity, we study the local behavior near the singular point $x^{*}=0$ assuming

$$
f_{i}(\alpha, 0)=0
$$

for all $i=0,1, \ldots, m$ and all considered $\alpha$. The linearized (bilinear) control system is

$$
\begin{aligned}
\dot{y}(t) & \left.=A_{0}(\alpha) y(t)+\sum_{i=1}^{m} u_{i}(t) A_{i}(\alpha) y(t)\right), \\
u & =\left(u_{i}\right) \in \mathcal{U},
\end{aligned}
$$

where $A_{i}(\alpha):=\frac{\partial}{\partial x} f_{i}(\alpha, 0)$ for all $i$. The corresponding Lyapunov exponents are given by

$$
\lambda\left(u, y_{0}\right)=\limsup _{t \rightarrow \infty} \frac{1}{t} \log \left|y^{\alpha}\left(t, y_{0}, u\right)\right|
$$

and the Lyapunov spectra are

$$
\Sigma^{\alpha}=\left\{\lambda\left(u, y_{0}\right), y_{0} \neq 0 \text { and } u \in \mathcal{U}\right\} .
$$

By Grünvogel (Grünvogel, 2000), it is known that, for fixed $\alpha$, the Lyapunov spectrum determines the controllability behavior near the origin (see below for a precise statement),

We model slowly varying $\alpha$ by requiring that $\dot{\alpha}(t)=\varepsilon>0$, small. In other words, we consider the systems in $\mathbb{R}^{d+1}$

$$
\begin{aligned}
\dot{x}(t) & =f_{0}(\alpha(t), x(t))+\sum_{i=1}^{m} u_{i}(t) f_{i}(\alpha(t), x(t)), \\
\dot{\alpha}(t) & =\varepsilon, \\
u & \in \mathcal{U} .
\end{aligned}
$$

Prescribing an initial value $a_{0}$ for $\alpha$ at time $t=0$, the equation in $\mathbb{R}^{d}$ can also be rewritten as the nonautonomous equation

$\dot{x}(t)=f_{0}\left(a_{0}+\varepsilon t, x(t)\right)+\sum_{i=1}^{m} u_{i}(t) f_{i}\left(a_{0}+\varepsilon t, x(t)\right)$.

The solutions of $(1.4)^{a_{0}, \varepsilon}$ are determined by the initial value $x(0)=x_{0} \in \mathbb{R}^{d}$ and the control 
$u$. We denote them by $\psi^{\varepsilon}\left(t, x_{0}, a_{0}, u\right)$ and the corresponding reachable sets are

$\mathcal{O}_{\leq T}^{a_{0}, \varepsilon_{+}+}(x)=\left\{\psi^{\varepsilon}\left(t, x_{0}, a_{0}, u\right), 0 \leq t \leq T, u \in \mathcal{U}\right\}$.

Note that these objects are defined for all $\varepsilon$ with $|E|$ small enough. Equation (1.3) describes a singularly perturbed system. Some results on the stability behavior and the Lyapunov spectrum of these systems are known; see, in particular, Grammel/Shi (Grammel and Shi, 2000), Vigodner (Vigodner, 1997), Artstein (Artstein, 1998). They are based on averaging techniques, which do not play a role in this paper. Instead we concentrate on control sets and their properties. See (Colonius and Kliemann, 2000) for an exposition of their basic properties.

\section{PRELIMINARIES AND PROBLEM FORMULATION}

In this section, we cite a result on the existence of control sets near singular points and formulate the corresponding problem for the singularly perturbed system (1.3)

The following result due to Grünvogel (Grünvogel, 2000 , Theorem 8.1 ) shows that control sets near the singular point are determined by the Lyapunov spectrum. Recall that a subset $D$ of $\mathbb{R}^{d}$ with nonvoid interior is a control set if it is a maximal set with the property $D \subset \operatorname{cl}\{\varphi(t, x, u), t>0$ and $u \in \mathcal{U}\}$ for all $x \in D$; here $\alpha \in A$ is fixed.

Theorem 1. Consider control system (1.1) and assume that

(i) there are periodic control functions $u^{s}$ and $u^{h}$ such that for $u^{s}$ the linearized system is exponentially stable, i.e.. the corresponding Lyapunov (Floquet) exponents satisfy

$$
0>\lambda_{1}^{s}>\ldots>\lambda_{d}^{s},
$$

and for $u^{h}$ the corresponding Lyapunov exponents satisfy

$$
\lambda_{1}^{h} \geq \ldots \geq \lambda_{k}^{h}>0>\lambda_{k+1}^{h}>\ldots>\lambda_{d}^{h}
$$

(ii) All pairs $\left(u^{h}, x\right) \in \mathcal{U} \times \mathbb{R}^{d}$ with $x \neq 0$ are strong inner pairs, i.e. $\mathcal{\tau}\left(t, x, u^{h}\right) \in \operatorname{int} \mathcal{O}^{a .+}(x)$ for all $t>0$ where $\mathcal{O}^{\alpha,+}(x):=\{r(\tau, x, u, a), \tau>0$ and $u \in \mathcal{U}\}$.

Then there exists a control set $D^{\alpha}$ with nonvoid interior such that $0 \in \partial D^{\alpha}$.

Remark 1. Grünvogel (Grünvogel, 2000) shows that there are no control sets in a neighborhood of the origin if zero is not in the interior of the Morse spectrum of the linearized ststem. This also follows from a Hartman-Grobman Theoren for skew product flows: see Bronstein/Kopanskii
(Bronstein and Kopanskii, 1994). One has to take into account, that the spectral condition implies hyperbolicity, since the base space $\mathcal{U}$ is chain recurrent. Then the use of appropriate cut-off functions yields the desired local version.

Remark 2. Assumption (i) in the previous theorem is in particular satisfied, if 0 is in the interior of the highest Floquet spectral interval and the corresponding subbundle is one-dimensional.

\section{DYNAMIC BIFURCATIONS}

In a number of control systems one observes numerically the following behavior: for some $\alpha$ values the singular point $x^{*}$ is exponentially stable for all controls, hence there are no control sets near $x^{*}$. Then, for increasing (constant) $\alpha$-values, control sets $D^{\alpha}$ occur with $x^{*} \in \partial D^{\alpha}$ and grow. For some upper $\alpha$-value, they move away from $x^{*}$. It is our aim to show that, for $\varepsilon>0$, small enough, also the resulting singularly perturbed system shows a similar controllability behavior. It is not obvious, however, how to formulate the desired property. Clearly, the resulting time dependent system has no control sets. On the other hand, only the component in $\mathbb{R}^{d}$ is of interest, and the parameter $\alpha(t)$ changes on a different time scale. We consider controllability only for the $\mathbb{R}^{d}$. component.

We will assume the following accessibility rank condition: Let

$$
\mathcal{L}^{\alpha}=\mathcal{L} \mathcal{A}\left\{f_{0}(\alpha, \cdot), \ldots, f_{m}(\alpha, \cdot)\right\}
$$

denote the Lie algebra generated by these vector fields, and for $x \in \mathbb{R}^{d}$ let $\Delta_{\mathcal{L}^{\circ}}(x)$ be the subspace of the tangent space $\mathbb{R}^{d}$ generated by the vector fields in $\mathcal{L}^{\alpha}$. The accessibility rank condition requires for all $\alpha$

$$
\operatorname{dim} \Delta_{\mathcal{L}^{\alpha}}(x)=d \text { for all } 0 \neq x \in \mathbb{R}^{d} .
$$

This implies local accessibility; that is, $\mathcal{O}_{\leq T}^{a}+(x)$ and $\mathcal{O}_{\leq T}^{\alpha,-}(x)$ have nonvoid interiors for all $T>0$ and all $0 \neq x \in \mathbb{R}^{d}$

Theorem 2. Assume that conditions (i) and (ii) in Theorem 1 are satisfied for all $\alpha \in A$. Consider an interval $I=\left(a_{0}, b_{0}\right) \subset A$. Then for every compact subset $Q \subset \bigcap_{\alpha \in I}$ int $D^{\alpha}$ there is $T_{0}>0$ with the following property: For all $x, z \in Q$ and for every $\xi>0$. small enough. there are a control function $u \in \mathcal{U}$ and a time $0<T<T_{0}$ such that $a_{0}+\varepsilon T \in I$ and

$$
\iota^{\varepsilon}\left(T, x, a_{0}, u\right)=z .
$$

Proof. It is clear that for $\bar{z}=0$ a subset $Q$ as above is contained in a control set for every $\alpha$ system. We have to show that the controllability property remains valid for small $s$. 
Let $x, z \in Q$ and $\alpha_{0} \in A$. As in the proof of Krener's theorem (see, e.g., (Colonius and Kliemann, 2000)), accessibility rank condition (3.1) implies that we may choose $u^{1} \ldots ., u^{d} \in$ $U$ and $T>0$ with the following properties: Consider the map $F:(0, T)^{d} \subset \mathbb{R}^{d} \rightarrow$ $\mathbb{R}^{d}: \quad:=\left(\tau_{1}, \ldots \tau_{d}\right) \mapsto \varphi\left(\sum_{i=1}^{d} \tau_{i}, x, u^{\tau}, a_{0}\right)$ where $u^{\tau} \in \mathcal{U}$ is a control with $u(t)=u^{i}$ for $t \in\left[\tau_{0}+\tau_{1}+\ldots+\tau_{i-1}, \tau_{0}+\tau_{1}+\ldots+\tau_{i}\right]$ and $\sum_{i=1}^{d} \tau_{i}=T$. Then the rank of $F$ equals $d$ at every $\tau$. Choose $T>0$ small enough such that $y_{1}:=\varphi\left(T, x, u^{\tau}, a_{0}\right) \in$ int $D^{\alpha_{0}}$. By definition,

$$
\varphi\left(T, x, u^{\top}, a_{0}\right)=\psi^{0}\left(T, x, a_{0}, u^{\tau}\right) .
$$

The implicit function theorem applied to the map $\mathbb{R} \times \mathbb{R}^{d} \rightarrow \mathbb{R}^{d}+\left(\varepsilon,\left(\tau_{1}, \ldots \tau_{n}\right)\right) \mapsto \psi^{\varepsilon}\left(\sum_{i=1}^{n} \tau_{i}, x, a_{0}, u^{\tau}\right)$ at $\varepsilon=0$ implies that there are $\delta_{1}>0$ and a neighborhood $N_{1}\left(y_{1}\right)$ with $N_{1}\left(y_{1}\right) \subset \mathcal{O}^{a_{0}, \varepsilon,+}(x)$ for all $\varepsilon<\delta_{1}$. There exists a time $S_{1}>0$ and a control $u_{1}$ such that $\varphi\left(S_{1}, y_{1}, u_{1}, a_{0}\right)=z$. With the same arguments as above one finds $y_{2}=$ $\varphi\left(-T_{2}, y_{1}, u_{2}^{\tau}, a_{0}\right)$ and a neighborhood $N_{2}\left(y_{2}\right) \subset$ $N_{1}\left(y_{1}\right)$ with $N_{2}\left(y_{2}\right) \subset \mathcal{O}^{a_{0}, \varepsilon,-}(z)$ for $\varepsilon<\delta_{2}$. Together, we have found $\delta_{0}:=\min \left(\delta_{1}, \delta_{2}\right)>0$ and the open set $N\left(y_{2}\right)$ such that $x$ can be steered into every point of $N\left(y_{2}\right)$ and every point in $N\left(y_{2}\right)$ can be steered into $z$ in the system with $\varepsilon<\delta_{0}$. Interchanging the roles of $x$ and $z$ and then using continuous dependence on initial values, we conclude that the compact set $Q$ can be covered by finitely many open sets where controllability in uniformly bounded time is possible for the systems with $\varepsilon>0$, small enough. This proves the assertion.

This result is still unsatisfactory, because the intersection $\bigcap_{\alpha \in I}$ int $D^{\alpha}$ may be small. Instead we will generalize the notion of control sets for the singularly perturbed control system (1.3). The control(lability) set is replaced by a family of subsets of the state space $\mathbb{R}^{d}$ depending on $a \in A$. which we call controllability bundles. or for short, control bundles.

Definition 1. Consider the singularly perturbed control system (1.3) A family of subsets $D^{\alpha} \subset$ $\mathbb{R}^{d}, a \in A$, of $\mathbb{R}^{d}$ with nonvoid interiors is called a control bundle, if for all $\alpha<\alpha^{\prime}$ in $A$ and all $x \in D^{\alpha}$ and all $y \in \operatorname{int} D^{\alpha^{\prime}}$ there are $\hat{s}_{0}>0$ and $T_{0}>0$ such that for all $0<\varepsilon<\varepsilon_{0}$ there are $0<$ $t<T_{0}$ and a control $u \in \mathcal{U}$ with $\iota^{, \varepsilon}(t, x, a, u)=y$. and the family $D^{\alpha}$ is maximal with this property. i.e. if a family of subsets $\left\{\tilde{D}^{\alpha}, a \in A\right\}$ satisfies these properties and $D^{\alpha} \subset \bar{D}^{\alpha}$ for all $a \in A$. then $D^{\alpha}=\tilde{D}^{\alpha}$ for all $\alpha \in A$.

Remark 3. It is tempting to define control bundles for general nonautonomous control systems by considering families of subsets $D^{t}$ of the state space depending on time $t$. Then, however. (ap- proximate) controllability from every point in $D^{t}$ to $D^{s}$ with $t<s$ is not appropriate; in the autonomous case it would reduce to controllability in arbitrarily short time. In the singularly perturbed situation we can extend time by reducing $\varepsilon$.

The following theorem is the main result of this paper.

Theorem 3. Consider a family of nonempty subsets $\left\{D^{\alpha}, \alpha \in A\right\}$ such that clint $D^{\alpha}=\operatorname{cl} D^{\alpha}$ for all $\alpha$ and the map $\alpha \mapsto \operatorname{cl} D^{\alpha}$ is continuous in the Hausdorff metric. Then the following properties are equivalent:

(i) For every $\alpha \in A$ the set $D^{\alpha}$ is a control set for system $(1.1)^{\alpha}$ with frozen parameter value $\alpha \in A$;

(ii) The sets $\left\{D^{\alpha}, \alpha \in A\right\}$ form a control bundle for singularly perturbed system (1.4).

Proof. Let $\left\{D^{\alpha}, \alpha \in A\right\}$ be a control bundle with the indicated properties. Then every set $D^{a_{0}}$ is a control set of the system with frozen parameter value $a_{0} \in A$. In fact,

$$
\bigcap_{\alpha \in\left[a_{0}, a_{0}+1 / n\right]} \operatorname{int} D^{\alpha}
$$

converges to $D^{\alpha_{0}}$ for $n \rightarrow \infty$. For $x, y$ in this intersection and $\varepsilon$ small enough for one finds controls $u^{\varepsilon}$ and times $t^{\varepsilon}<T_{0}$ with $\psi^{\varepsilon}\left(t^{\varepsilon}, x, a_{0}, u^{\varepsilon}\right)=y$. By compactness of $\mathcal{U}$ in the weak ${ }^{*}$ topology (compare (Colonius and Kliemann, 2000)), boundedness of $t^{\varepsilon}$ and continuity, cluster points $t$ and $u$ for $\varepsilon \rightarrow 0$ satisfy

$$
\varphi\left(t, x, u, a_{0}\right)=\psi^{0}\left(t, x, a_{0}, u\right)=y .
$$

Conversely, consider a family $\left\{D^{\alpha} \cdot \alpha \in A\right\}$ such that $D^{\alpha}$ are control sets for system $(1.1)^{\alpha}$ and the map $\alpha \mapsto \operatorname{cl} D^{\alpha}$ is continuous. Fix $\alpha<\alpha^{\prime}$ in $A$ and points $x \in \operatorname{int} D^{\alpha}$ and $y \in \operatorname{int} D^{\alpha^{\prime}}$. By continuous dependence on the parameter there are finitely many points

$$
\alpha_{0}=\alpha<\alpha_{1}<\ldots<\alpha_{m}=\alpha^{\prime}
$$

with

$$
x \in \bigcap_{\alpha \in\left[\alpha_{0} . \alpha_{1}\right]} \operatorname{int} D^{\alpha}
$$

and

$$
\bigcap_{\alpha \in\left[\alpha_{j}, a_{j-1}\right]} \operatorname{int} D^{\circ} \neq \emptyset \text { for all } j=1 \ldots . m-1 \text {.. }
$$

Thus there are finitely many points

$$
\begin{gathered}
x_{0}=x \in \operatorname{int} D^{\alpha} . x_{1} \in \operatorname{int} D^{\alpha_{1}} . \ldots . \\
x_{m-1} \in \operatorname{int} D^{\alpha_{m-1}} . x_{m}=y \in \operatorname{int} D^{\alpha_{m}}
\end{gathered}
$$

with

$$
x_{j} \in \bigcap_{\alpha \in\left[\alpha_{1}, \alpha_{j-i}\right]} \operatorname{int} D^{\alpha} \text { for all } j \text {. }
$$


Now we apply Theorem 2 on each of the finitely many intervals $\left[\alpha_{j}, \alpha_{j+1}\right]$ and obtain numbers $\varepsilon_{0}, \varepsilon_{1}, \ldots, \varepsilon_{m-1}>0$ such that $x_{j}$ can be steered to $x_{j+1}$ for all $0<\varepsilon<\varepsilon_{j}$. Taking $0<\varepsilon<$ $\max \left\{\varepsilon_{j}, j=0, \ldots, m-1\right\}$ we conclude that we can steer the point $x=x_{0}$ to $y=x_{m}$. Thus the family $\left\{\operatorname{int} D^{\alpha}, \alpha \in A\right\}$ satisfies the controllability property required in the definition of control bundles. Hence there exists a control bundle containing these sets. It must coincide with $\left\{D^{\alpha}, \alpha \in A\right\}$ since its sets are control sets containing $D^{\alpha}$

Remark 4. A control set depends continuously on parameters, if its closure coincides with the chain control set containing it; compare (Colonius and Kliemann, 2000, Corollary 3.4.7).

\section{CONCLUSIONS}

We have seen how controllability properties of dynamically perturbed systems are inherited from the corresponding systems with frozen parameter values. It appears possible to generalize at least part of the arguments to more general singularly perturbed systems, where instead of $\dot{\alpha}=\varepsilon$ one has a slow system of the form

$$
\dot{z}(t)=\varepsilon g(z(t), u(t)) \text { in } \mathbb{R}^{k}
$$

with an appropriate vector field $g$ on $\mathbb{R}^{k}$.

\section{REFERENCES}

Artstein, Z. (1998). Stability in the presence of singular perturbations. Nonlinear Analysis, TMA 34, 817-827.

Bronstein, I. U. and A. Ya. Kopanskii (1994). Smooth Invariant Manifolds and Normal Forms. World Scientific.

Colonius, F. and W. Kliemann (2000). The Dynamics of Control. Birkhäuser.

Grammel, G. and P. Shi (2000). On the asymptotics of the Lyapunov spectrum under singular perturbations. IEEE Trans. Automatic Control 45, 565-568.

Grünvogel, S.M. (2000). Lyapunov Spectrum and Control Sets. Dissertation, Universität Augsburg, Augsburger Mathematische Schriften no, 34, Wißner Verlag, Augsburg.

Vigodner, A. (1997). Limits of singularly perturbed control problems with statistical dynamics of fast motions. SIAM J. Control Optim. 35, 1-28. 